\title{
Dengue Knowledge and Preventive Practices among Rural Residents in Samar Province, Philippines
}

\author{
Begonia C. Yboa, Leodoro J. Labrague* \\ College of Nursing \& Health Sciences, Samar State University, Philippines \\ *Corresponding author: Leo7_ci@yahoo.com
}

Received March 02, 2013; Revised April 05, 2013; Accepted April 08, 2013

\begin{abstract}
The World Health Organization (WHO) classifies dengue as a disease important in public health. The epidemiology and ecology of dengue infections are strongly associated with human habits and activities. The present study aimed to evaluate the knowledge and practices regarding dengue infections among rural residents in Samar Province, Philippines. A cross sectional design was adopted for this investigation. Convenience samples of six hundred forty six (646) residents who were visiting the rural health units in different municipalities of Samar, Philippines were taken as participants in study. More than half of the respondents had good knowledge (61.45\%) on causes, signs and symptoms, mode of transmission, and preventive measures about dengue. More than half of the respondents used dengue preventive measures such as fans $(n=340,52.63 \%)$, mosquito coil $(n=458,70.90 \%)$, and bed nets $(n=387,59.91 \%)$ to reduce mosquitoes while only about one third utilized insecticides sprays $(n=204$, $31.58 \%)$ and screen windows $(n=233,36.07 \%)$ and a little portion used professional pest control $(n=146,22.60 \%)$. There was no correlation between knowledge about dengue and preventive practices $(\mathrm{p}=0.75)$. Television/Radio was cited as the main source of information on dengue infections. Findings suggest that better knowledge does not necessarily lead to better practice of dengue measures. Educational campaigns should give more emphasis dengue transmissions and on cost effective ways of reducing mosquito and preventing dengue such as environmental measures and control. Furthermore, wide range of information, skills and support must be provided by the government to increase dengue awareness among residents.
\end{abstract}

Keywords: dengue fever, dengue preventive practices, dengue knowledge, Samar Province

\section{Introduction}

Dengue fever (DF) is a mosquito-borne viral infection causing a severe flu-like illness and, sometimes causing a potentially lethal complication called severe dengue transmitted by bites of Aedes aegypti and Aedes albopictus mosquito [1]. Dengue fever (DF) is caused by any of four closely related viruses, or serotypes: DENV 1 , DENV 2, DENV 3, and DENV 4. Symptoms of infection is characterized by a sudden onset of high fever (103$106^{\circ} \mathrm{F}$ ), severe headache, backache, intense pain in joints and muscles, retro-orbital pain, nausea and vomiting and a generalized erythematous rash that usually begin 4-7 days after the mosquito bite and typically last 3-10 days [2]. However, infection with a dengue virus serotype can also produce a more complex and severe form of clinical manifestations like hemorrhage and shock.

In the recent years dengue fever has become international global public health concern as there has a dramatic increase of cases of dengue in tropical and subtropical regions around the world, predominantly in urban and semi-urban areas. According to the World Health Organization, dengue fever in its severest form is a leading cause of serious illness and death among children in some Asian and Latin American countries - is endemic in more than 100 countries. It is estimated that 50-
500,000 cases of dengue fever occur worldwide [2,3]. Out of the 2.5 billion people at risk globally; about 1.8 billion or more than 70 percent of them live in the Asia-Pacific region [4]. Emergence of dengue could be the result of growing levels of urbanization, international trade and travel which disseminate both vector and viruses [5].

The first confirmed epidemic of dengue fever was recorded in the Philippines in 1953-1954. Since then, several strategies had been formulated to contain the spread and increasing incidence of dengue [20]. In the recent years, significant numbers of dengue cases were recorded in the Philippines. The National Epidemiology Center of the Philippines' Department of Health reports a total of 132,046 dengue cases from January to 13 October 2012. This is $24.92 \%$ higher compared to the same time period in 2011. Of the total cases, $20.42 \%$ came from the National Capital Region (NCR), with highest contributors from Quezon City (7 754 cases), Manila (4 379 cases) and Caloocan City (2 967 cases). Next to NCR, Region III and Region IV-A registered the highest number of cases, which are $15.79 \%$ and $15.66 \%$ respectively, to the overall figure $[6,22]$.

The World Health Organization and Centers for Disease Control and Prevention (CDCP) recommends extensive community educational campaigns that emphasize reducing vector breeding sites as an effective way of dengue prevention $[12,14]$. This recommendation is supported by various researches showing that 
community education can be more effective in reducing dengue vector breeding sites than chemicals alone [13]. Several studies suggest that better knowledge of dengue and dengue vector prevention practices among people was one of the predictors of better practices of dengue prevention $[7,8,9,10]$. Van Benthem (2002) claimed that people with higher knowledge on dengue reported a significantly higher use of prevention measure than people with low knowledge [7]. Meanwhile, Chusongsang (2005) reported that household leaders with high level of knowledge had better dengue prevention practices [8] Itrat and Colleagues, (2008) also found that preventive practices regarding dengue were consistent with the knowledge about these practices [9]. However, other studies conducted did not yield the same result. Knowledge on dengue did not necessarily translated to dengue preventive practices $[11,15,18]$.

In the Philippines, despite of the extensive campaign of the government against dengue, there are evidences of increasing rates of dengue morbidity in the recent year [6]. Moreover, as to authors' knowledge, no empirical data investigating knowledge and practices on dengue prevention within the Province of Samar, Philippines exists. Further, recent outbreaks of dengue in the province necessitated development of this study. The knowledge that could be gained in this investigation would guide public administrators to plan, design and initiate initiatives, programs, and policies relative to dengue prevention which could be used to address the ever growing problems on dengue fever infections.

\section{Research Objectives}

This investigation was undertaken to evaluate the knowledge and practices regarding dengue infections among rural residents in Samar Province, Philippines.

\section{Methodology}

A cross sectional study was adopted for this investigation among the different types of descriptive studies. This study design is appropriate as the main objective of this investigation was to assess the knowledge and practices regarding dengue infections among rural residents.

\subsection{Participants}

Data were collected over a period of three (3) months from September to February, 2012. A convenience sample of six hundred forty six (646) residents aged 18 years or above who were visiting the rural health units in different municipalities of Samar, Philippines were all provided the opportunity to be respondents in the investigation.

\subsection{Measures}

To gather data, the investigators utilized the questionnaires developed by Shuaib et al [11] with modifications. Questions were based on causes, signs and symptoms of dengue, transmission modes, attitude towards dengue; and dengue preventive practices covering 25 items, with possible responses of 'yes' and 'no'. 'Yes' is given a value of 1 point, and 'no' with 0 points; the maximum possible score is 10 . The higher the score, the greater the assumed knowledge about dengue and dengue prevention the respondent has. Result of test was interpreted as follows; $21-25$ as "Excellent Knowledge", $16-20$ as "Good Knowledge", $11-15$ as "Moderate Knowledge", $6-10$ as "Fair Knowledge", and $0-5$ as "Poor Knowledge".

Part II of the questionnaire was used to evaluate the dengue prevention practices. There are 12 indicators with a scale of $0-4$ points: $0=$ never, $1=$ seldom, $2=$ sometimes, 3 = usually, and $4=$ always, giving a score range of $0-48$. In determining the extent of practice, the following scaling was used; for Very Great Extent $=4.51$ 5.00, Great Extent $=3.51-4.50$, Moderately Extent $=2.51$ 3.50 , Limited Extent $=1.51-2.50$, and Not at all $=1.00$ 1.50. The higher the mean score, the better that person carries out the dengue prevention practices.

The questionnaire was validated for its reliability resulting in statistical value of 0.90 (Cronbach's alpha). The questionnaire and was drafted in a structured format and was pilot tested before distributed to the respondents enrolled in this investigation. Refinement and modifications were done on the basis of pretest results. Furthermore, questionnaires were validated through expert validation by five experts in the field of infectious diseases. The questionnaires were handed out by the investigators at the site personally, and collected on the spot once they have been completed individually and anonymously by the respondents.

\subsection{Ethical Considerations}

The study protocol was reviewed and approved by the Health Ethics Committee of Samar State University, Philippines. All the participants were fully informed about the purpose of the study. Confidentiality of the respondents was maintained only by a code number on the questionnaire.

\subsection{Data Analysis}

The data from the questionnaire were coded and entered into a computerized data base and analyzed using SPSS, version 19. Frequencies and percentages were used for analyzing the selected socio- demographic data while mean and median were used to assess responses of the respondents on the questionnaire. Pearson's correlation coefficient was utilized to test the relationships between knowledge and dengue preventive practices, while Fisher's t test was utilized to determine significance of correlations. A p-value of equal to or less than 0.05 was considered statistically significant.

\section{Results}

A total of six hundred forty six (646) respondents were recruited to participate in the investigation consisting of $319(49.38 \%)$ male and $327(50.62 \%)$ female. Majority of the respondents belong to the age group of 18 to 23 years old $(n=394,60.99 \%)$ and not married $(n=458,70.89 \%)$. As to education, about half of the respondents were college undergraduate $(\mathrm{n}=331,51.24 \%)$ and have a family monthly income of less than $\$ 125 /$ month $(n=447$, $69.19 \%)$. 
Table 1. Demographic Characteristics of the Respondents

\begin{tabular}{|c|c|c|c|}
\hline \multicolumn{2}{|c|}{ Characteristics } & Frequency & Percentage \\
\hline \multirow{2}{*}{ Gender } & Male & 327 & 50.62 \\
\hline & Female & 319 & 49.38 \\
\hline \multirow{8}{*}{ Age (in years) } & $18-23$ & 394 & 60.99 \\
\hline & $24-29$ & 84 & 13.00 \\
\hline & $30-35$ & 34 & 5.26 \\
\hline & $36-41$ & 33 & 5.11 \\
\hline & $42-47$ & 31 & 4.79 \\
\hline & $48-53$ & 36 & 5.57 \\
\hline & $54-59$ & 16 & 2.47 \\
\hline & 60 and Above & 18 & 2.78 \\
\hline \multirow{4}{*}{ Marital Status } & Single & 458 & 70.89 \\
\hline & Married & 160 & 24.76 \\
\hline & Widowed/Widower & 15 & 2.32 \\
\hline & Separated & 13 & 2.01 \\
\hline \multirow{6}{*}{ Education } & College Graduate & 135 & 20.89 \\
\hline & College Undergraduate & 331 & 51.24 \\
\hline & High School Graduate & 99 & 15.32 \\
\hline & High School Undergraduate & 53 & 8.20 \\
\hline & Elementary Graduate & 12 & 1.86 \\
\hline & Elementary Undergraduate & 16 & 2.48 \\
\hline \multirow{7}{*}{ Monthly Income } & $\$ 751$ and above & 15 & 2.32 \\
\hline & $\$ 626-\$ 750$ & 43 & 6.65 \\
\hline & $\$ 501-\$ 625$ & 15 & 2.32 \\
\hline & $\$ 376-\$ 500$ & 24 & 3.71 \\
\hline & $\$ 250-\$ 375$ & 33 & 2.32 \\
\hline & $\$ 126-\$ 250$ & 69 & 10.68 \\
\hline & $\$ 125$ and below & 447 & 69.19 \\
\hline
\end{tabular}

Table 2. Responses to Knowledge on Dengue Infections Questionnaire

\begin{tabular}{|c|c|c|c|c|}
\hline Statements & Yes & $\%$ & No & $\%$ \\
\hline \multicolumn{5}{|l|}{ Knowledge on the cause } \\
\hline Dengue is caused by a mosquito bite. & 600 & 92.87 & 46 & 7.12 \\
\hline Dengue mosquitoes likely to feed/bite in the afternoon. & 451 & 69.81 & 195 & 30.18 \\
\hline \multicolumn{5}{|l|}{ Knowledge of symptoms } \\
\hline Fever is a symptom of dengue. & 606 & 93.8 & 40 & 6.19 \\
\hline Headache is a symptom of dengue fever. & 564 & 87.3 & 82 & 12.69 \\
\hline Joint pains are symptoms of dengue fever. & 525 & 81.26 & 121 & 18.73 \\
\hline Muscle pain is a symptom of dengue fever. & 462 & 71.51 & 184 & 28.48 \\
\hline Pain behind the eyes is a symptom of dengue fever & 253 & 39.16 & 393 & 60.83 \\
\hline Rashes are symptom of dengue fever. & 524 & 81.11 & 122 & 18.88 \\
\hline Abdominal pain is a symptom of dengue fever. & 531 & 82.19 & 115 & 17.8 \\
\hline \multicolumn{5}{|l|}{ Knowledge of transmission } \\
\hline Flies transmit Dengue fever. & 234 & 36.22 & 412 & 63.77 \\
\hline Ticks transmit Dengue fever. & 340 & 52.63 & 306 & 47.36 \\
\hline All types of mosquitoes transmit Dengue fever. & 310 & 47.98 & 336 & 52.02 \\
\hline Aedes mosquito transmits Dengue Fever. & 519 & 80.34 & 127 & 19.65 \\
\hline Person to person contact transmits Dengue fever. & 291 & 45.04 & 355 & 54.95 \\
\hline Dengue fever can be transmitted by a blood transfusion. & 490 & 75.85 & 156 & 24.14 \\
\hline Dengue fever can be transmitted by a needle stick. & 463 & 71.67 & 183 & 28.32 \\
\hline Dengue fever can be transmitted by sexual intercourse. & 210 & 32.5 & 436 & 67.49 \\
\hline \multicolumn{5}{|l|}{ Knowledge on Dengue Prevention } \\
\hline Mosquitoes breed in standing water. & 398 & 61.6 & 248 & 38.39 \\
\hline Window screens and bed nets reduce mosquitoes & 556 & 86.06 & 90 & 13.93 \\
\hline Insecticide sprays reduce mosquitoes and prevent dengue & 609 & 94.27 & 37 & 5.72 \\
\hline Covering water containers reduce mosquitoes & 623 & 96.43 & 23 & 3.56 \\
\hline Removal of standing water can prevent mosquito breeding & 583 & 90.24 & 63 & 9.75 \\
\hline Mosquito repellants prevent mosquitoes & 601 & 93.03 & 45 & 6.96 \\
\hline Cutting down bushes can reduce mosquitoes and dengue & 553 & 85.6 & 93 & 1.43 \\
\hline Pouring chemicals in standing water can kill mosquito larvae & 558 & 86.37 & 88 & 13.62 \\
\hline
\end{tabular}

Vast majority of the respondents knew that dengue is caused by a mosquito bite $(\mathrm{n}=600,92.87 \%)$ and it is more likely to feed/bite in the afternoon $(n=451,69.81 \%)$. As to symptoms, most of the respondents agreed that a person with dengue infections may develop typical symptoms like fever, headache, joint pains, muscle pain, rashes, and abdominal pain. Only $39.16 \%$ or 253 of the respondents knew that pain behind the eyes is a symptom of dengue infections. With regards to dengue transmission, $80.34 \%$ or 519 believed that aedes mosquito transmits dengue infection; however, a significant number of respondents claimed that flies, ticks, and all types of 
mosquitoes also transmit dengue infections. About one fourth of the respondents believed that dengue infection may be transmitted by blood transmission $(n=490$, $75.85 \%)$ and needle stick injury $(\mathrm{n}=463,71.67 \%)$ while $32.5 \%$ or 210 respondents claimed that it can be transmitted through sexual intercourse. As to knowledge on dengue prevention, greater proportion of the respondents cited the use of window screen, bed nets, insecticide sprays, covering of water, removal of standing water, cutting down bushes, and pouring chemicals in standing water as measures to reduce mosquitoes and dengue (Table 2).

Table 3. Respondents Knowledge on Dengue Infection

\begin{tabular}{|c|c|c|}
\hline Score Range & Frequency & Percentage (\%) \\
\hline $21-25$ & 195 & 30.18 \\
\hline $16-20$ & 397 & 61.45 \\
\hline $11-15$ & 54 & 8.36 \\
\hline $6-10$ & 0 & 0 \\
\hline $0-5$ & 0 & 0 \\
\hline Average Score & \multicolumn{2}{|c|}{19.01} \\
\hline
\end{tabular}

Table 3 reflects the cumulative scores of the respondents on the questionnaires on dengue infections. More than half $(61.45 \%)$ of the respondents scored within the score range of 16 to 20 which is interpreted as "Good
Knowledge", while $30.18 \%$ scored within the score range of 21 to 25, which is interpreted as " Very Good Knowledge". In general, respondents possess "Good Knowledge" on dengue infections with a weighted mean score of 19.01 .

Table 4 portrays participants' self-reported practices toward dengue infections prevention and control. As gleaned on the table, most of the respondents undertook measures to reduce mosquitoes and prevent dengue. For instance, more than half of the respondents used fans $(\mathrm{n}=$ $340,52.63 \%)$, mosquito coil $(\mathrm{n}=458,70.90 \%)$, and bed nets $(\mathrm{n}=387,59.91 \%)$ to reduce mosquitoes while only about one third utilized insecticides sprays $(\mathrm{n}=204$, $31.58 \%)$ and screen windows $(n=233,36.07 \%)$. Only a little portion of the respondents used professional pest control $(n=146,22.60 \%)$ as means to reduce mosquitoes however, more than half of them employed environmental control measures such as eliminating standing water around house $(n=357,55.26 \%)$, cutting down of bushes in the yard $(n=468,72.45 \%)$, covering of water containers at home $(n=404,62.54 \%)$, and cleaning water filled containers around house ( $\mathrm{n}=414,64.09 \%)$. In general, respondents practiced dengue prevention to a "greater extent" with a grand mean of 3.02.

Table 4. Responses to Dengue Prevention Practices Questionnaire

\begin{tabular}{|c|c|c|c|c|c|c|c|c|c|c|c|}
\hline \multirow[b]{2}{*}{ Statements } & \multicolumn{11}{|c|}{ Extent of Practice } \\
\hline & 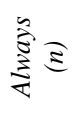 & $\delta^{\circ}$ & 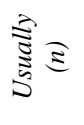 & $\delta^{\circ}$ & 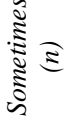 & o & 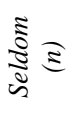 & $\delta^{\circ}$ & 高 & $0^{\circ}$ & 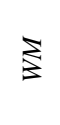 \\
\hline $\begin{array}{l}\text { Uses insecticide sprays to reduce } \\
\text { mosquitoes. }\end{array}$ & 204 & 31.58 & 121 & 18.73 & 175 & 27.09 & 62 & 9.60 & 84 & 13.00 & 2.45 \\
\hline $\begin{array}{l}\text { Uses professional pest control to reduce } \\
\text { mosquitoes. }\end{array}$ & 146 & 22.60 & 123 & 19.04 & 138 & 21.36 & 95 & 14.71 & 144 & 22.29 & 2.05 \\
\hline $\begin{array}{l}\text { Uses screen windows to reduce } \\
\text { mosquitoes. }\end{array}$ & 233 & 36.07 & 115 & 17.80 & 106 & 16.41 & 82 & 12.69 & 110 & 17.03 & 3.08 \\
\hline Uses fans to reduce mosquitoes. & 340 & 52.63 & 125 & 19.35 & 101 & 15.63 & 32 & 4.95 & 48 & 7.43 & 3.04 \\
\hline Uses bed nets to reduce mosquitoes. & 387 & 59.91 & 105 & 16.25 & 66 & 10.22 & 33 & 5.11 & 55 & 8.51 & 3.14 \\
\hline $\begin{array}{l}\text { Eliminates standing water around the } \\
\text { house to reduce mosquitoes. }\end{array}$ & 357 & 55.26 & 112 & 17.34 & 69 & 10.68 & 50 & 7.74 & 58 & 8.98 & 3.02 \\
\hline $\begin{array}{l}\text { Cuts down bushes in the yard to reduce } \\
\text { mosquitoes. }\end{array}$ & 468 & 72.45 & 82 & 12.69 & 49 & 7.59 & 27 & 4.18 & 20 & 3.10 & 3.46 \\
\hline $\begin{array}{c}\text { Uses mosquito coils to reduce } \\
\text { mosquitoes. }\end{array}$ & 458 & 70.90 & 86 & 13.31 & 60 & 9.29 & 25 & 3.87 & 17 & 2.63 & 3.46 \\
\hline Covers water containers in the home. & 404 & 62.54 & 101 & 15.63 & 57 & 8.82 & 42 & 6.50 & 42 & 6.50 & 3.21 \\
\hline $\begin{array}{l}\text { Cleans water filled containers and } \\
\text { ditches around the house. }\end{array}$ & 414 & 64.09 & 99 & 15.33 & 56 & 8.67 & 43 & 6.66 & 34 & 5.26 & 3.26 \\
\hline
\end{tabular}

Table 5 demonstrates respondents' sources of information about dengue. Majority of the respondents or $73.37 \%$ cited Television/Radio as the main source of information on dengue infections. In addition, few participants obtained such information from health workers and schools.

Table 5. Sources of Information Relative to Dengue Infections

\begin{tabular}{|c|c|c|}
\hline Sources of Information & Frequency & Percentage \\
\hline TV/Radio & 474 & 73.37 \\
\hline School & 32 & 4.95 \\
\hline Health workers & 33 & 5.11 \\
\hline Health Centers & 18 & 2.78 \\
\hline Hospitals & 19 & 2.94 \\
\hline Neighbors & 21 & 3.25 \\
\hline Brochures & 1 & 0.15 \\
\hline Newspaper & 2 & 0.31 \\
\hline Others & 5 & 0.77 \\
\hline
\end{tabular}

Relationship between respondents' knowledge and practices towards dengue infections posted an r-value of 0.0121 with a computed p-value of 0.7571 .

\section{Discussion}

This investigation evaluated the knowledge and preventive practices regarding dengue infections among rural residents in Samar Province, Philippines. The results of this investigation have demonstrated that respondents were knowledgeable of the concepts of dengue. When compared with previous studies conducted in Pakistan [9], Jamaica [11], Saudi [17], and Sri Lanka [23], knowledge on dengue in Filipino population appears to be high. However, the result of this study is in keeping with the results obtained in Brazil [15], Thailand [16], and India. [19] This may be due to intensified dengue awareness 
campaign efforts of the Philippine government to raise the people's level of awareness and knowledge on dengue.

It is essential to note that while majority of the respondents were highly informed of the causes, symptoms, and dengue preventive measures, still quite significant proportion of the respondents hold wrong notions about dengue transmissions. For instance, most of the respondents were aware that dengue is caused by mosquito bite and a person with dengue may manifests symptoms like fever, headache, joint and muscle pains, rashes, and abdominal pain. However, about half of the respondents believed that flies, ticks, and all types of mosquitoes transmit dengue while only one fourth of the respondents knew that pain behind is also a symptom of dengue. In a Jamaican study, most participants were aware that flies and ticks do not transmit dengue fever $(66.5 \%$ and $71.8 \%$ respectively) [11]. Good knowledge on the mosquito vector and signs and symptoms of dengue is essential in identifying the disease and in seeking early and appropriate medical treatment to save lives.

Surprisingly, about $30.18 \%$ of respondents were unaware that dengue mosquitoes are more likely to bite in the afternoon. According to World Health Organization (WHO), Aedes mosquito usually bites during the day [1]. Moreover, $38.39 \%$ were unaware that dengue mosquitoes breed in standing water. Bridging this gap in knowledge is important in planning and designing programs and activities to educate rural residents on preventive measures to combat dengue.

Findings also indicated that radio/television were the most cited sources of information on dengue infection relating with data gathered in from Pakistan [9], Malaysia [10], Jamaica [11], Thailand [16], India [19], and Sri Lanka [23]. Interestingly, only a few proportion of the respondents cited schools and health centers as sources of dengue information. In other countries, public health education programs have clearly proven its efficacy in increasing the knowledge and awareness of the disease. Thus, government can maximize the potential use of these educational and health institutions by providing adequate support like information, education and communication (IECs) materials and other visual aids that may effectively communicate dengue preventive measures.

Another important finding of this investigation was the high utilization of dengue preventive measures such as the use of fans, bed nets, mosquito coils and other control measures. However, only a little portion of the respondents utilize insecticide sprays, professional pest control, and screen windows as ways to reduce mosquito and prevent dengue. These strategies may be considered as costly considering that most of the respondents have limited financial capabilities. This suggests that governments' educational campaigns should give more emphasis on cost effective ways of preventing dengue such as environmental measures and control.

Key finding of this investigation was the insignificant relationship between knowledge about dengue and preventive practices. Knowledge about dengue fever did not necessarily translate to improve preventive measures. This result is inconsistent with previous studies conducted in Pakistan [9], and Malaysia [10] suggesting that knowledge alone is not a predictor of good practice. However, similar result was obtained in Jamaica [11], Brazil [15], and Thailand [18]. Better knowledge does not necessarily lead to better practice, most likely because people tend to stop doing good practice especially if no continuous monitoring is done.

While these data generated from this investigation are important as it is the first analysis conducted in the Province, nevertheless it has some limitations. The sampling design may have potentially limited the generalizability of the result since they were recruited based of researchers' convenience only and no rigid sampling was done. This investigation was conducted among residents in selected municipalities only. Exclusion of residents from other municipalities may have also affected the generalizability of the result.

Despite of some limitations posed by this investigation, the results provided useful inputs and knowledge that would guide government officials in planning, designing and initiating programs, and activities relative to dengue prevention which could be used to address the ever growing problems on dengue fever.

\section{Conclusions}

It could be inferred from this investigation that the level of knowledge about dengue and preventive practices among the study population is rather high. However, they face challenges such as greater access to correct information on dengue. In view of this result, government agencies and other non-government organizations should strengthen its programs on massive educational campaign to increase awareness and knowledge regarding dengue and preventive measures to reduce mosquito and prevent dengue. Information, education and communication (IECs) materials maybe provided in areas like schools and health centers making it more accessible for the residents to obtain. Knowledge of dengue, the vectors and transmission of disease may be incorporated into the school curriculum especially in areas where dengue is highly prevalent. Intersectoral coordination meetings should be conducted to identify possible partners for public education dengue control campaigns to help finance the program/activities. Reorientation training of community health workers should be conducted regularly to improve their technical skills and capability, and their ability to supervise prevention and control activities.

\section{Acknowledgement}

Investigators would like to thank all the participants for their time and effort in responding to the questionnaires.

\section{Conflict of Interest}

The author(s) declare that they have no competing interests.

\section{References}

[1] Heymann DL. Control of communicable disease manual. Eighteenth ed. Washington, DC: American Public Association; 2004. pp. 146-52. 
[2] World Health Organization (2009) Dengue Guidelines for Diagnosis, Treatment, Prevention, and Control: New Edition. Genev, 2004.

[3] World Health Organization. Media Centre. Dengue and severe dengue. Geneva, World Health Organization, 2008 (http://www.who.int/mediacentre/factsheets/fs117/en/).

[4] Guzman MG, Kouri G. Dengue and dengue hemorrhagic fever in the Americas: lessons and challenges. Journal of Clinical Virology $2003 ; 27: 1-13$.

[5] Egger JR, Ooi EE, Kelly DW,Woolhouse ME, Daviesa CR, ColemanaPG. Reconstructing historical changes in the force of infection of dengue fever in Singapore: implications for surveillance and control. Bulletin of the World Health Organization 2008; 86:187-96.

[6] Department of Health, National Epidemiology Center Public Health Surveillance and Informatics Division Disease Surveillance Report. Retrieved September 2, 2012.

[7] Van Benthem BH, Khantikul N, Kessels PJ, Somboon P, and Oskam L. Knowlegde and use of prevention measures related to dengue in North Thailand. Tropical Medicine of Int. Health. 2002;7(11), 993-1000.

[8] Chusongsung P. Factors affecting dengue hemorrhagic fever prevention and control behaviors of household leaders and primary school teachers in Kuan Khanun District, Phatthalung Province. Masters Thesis Faculty of Graduate studies, Chulalongkorn University). 2005.

[9] Itrat A, Khan A, Javaid S, Kamal M, Khan H, Javed S, et al Knowledge, awareness and practices regarding dengue fever among the adult population of dengue hit cosmopolitan. PLoS One 2008; 9(3):e2620.

[10] Hairi F, Ong CH, Suhaimi A, Tsung TW, bin Anis Ahmad MA, Sundaraj C, et al. A knowledge, attitude and practices (KAP) study on dengue among selected rural communities in the Kuala Kangsar district. Asia-Pacific Journal of Public Health 2003; 15:37-43

[11] Shuaib F, Todd D, Campbell-Stennett D, Ehiri J, Jolly P. Knowledge, attitudes and practices regarding dengue infection in Westmoreland, Jamaica. West Indian Med J. 2012; 59(2): 139-146.

[12] Center for Disease Control. Dengue fever. Colorado: CDC; 2005. [updated January 13, 2005; cited October 18, 2008]; Available from: http://www.cdc.gov/ncidod/dvbid/dengue/dengue-qa.htm.
[13] Espinoza-Gómez F, Hernández-Suárez C, Coll-Cárdenas R. Educational campaign versus malathion spraying for the control of Aedes aegypti in Colima, Mexico. Journal of Epidemiology and Community Health 2002; 56(2):148-52.

[14] World Health Organization. Comprehensive Guidelines for Prevention and Control of Dengue and Dengue Hemorrhagic Fever. Revised and Expanded Edition. Regional Office for South East Asia. 2011. (http://203.90.70.117/PDS_DOCS/B4751.pdf).

[15] Lenita Barreto Lorena Claro, Hélia Kawa, Luciana Tricai Cavalini and Maria Luiza Garcia Rosa. Community Participation in Dengue Control in Brazil. Dengue Bulletin, 2006; 30:214-222.

[16] Swaddiwudhipong W, Lerdlukanavonge P, Khumklam P, Koonchote S, Nguntra P, Chaovakiratipong C. A survey of knowledge, attitude and practice of the prevention of dengue hemorrhagic fever in an urban community of Thailand. The Southeast Asian Journal of Tropical Medicine and Public Health 1992;23:20711.

[17] Nahla Khamis Ragab Ibrahim*, Adnan Al-Bar, Mohamed Kordey, Ali Al-Fakeeh. Knowledge, attitudes, and practices relating to Dengue fever among females in Jeddah high Schools. Journal of Infection and Public Health, 2009; 2: 3040.

[18] Koenraadt CJ, Tuiten W, Sithiprasasna R, Kijchalao U, Jones JW, Scott TW. Dengue knowledge and practices and their impact on Aedes aegypti populations in Kamphaeng Phet, Thailand. The American Journal of Tropical Medicine and Hygiene 2006; 74:692-700.

[19] Anita Acharya, K. Goswami, S. Srinath \& A. Goswami. Awareness about dengue syndrome and related preventive practices amongst residents of an urban resettlement colony of south Delhi. J Vect Borne Dis, September 2005; 42(3):122-127.

[20] Gubler DJ. Dengue and dengue haemorrhagic fever. Clinical Microbiology Reviews 1998; 11: 480-496.

[21] Centers for Disease Control and prevention. Dengue Homepage. [Internet] http://www.cdc.gov/dengue/epidemiology/index.html Accessed: February 25, 2013.

[22] World Health Organization Western Pacific Region. [Internet] http://www.wpro.who.int/philippines/areas/communicable_disease s/dengue/en/. Accessed: February 25, 2013.

[23] Gunasekara, T. D. C. P., Velathanthiri, V. G. N. S., Weerasekara, M. M., Fernando, S. S. N., Peelawattage, M., Guruge, D., \& Fernando, S. Knowledge, attitudes and practices regarding dengue fever in a suburban community in Sri Lanka. Galle Medical Journal, 2012; 17(1), 10-17. 